\title{
Proton decay suppression in a supersymmetric SO(10) model
}

\section{Xiaojia Li and Da-Xin Zhang}

School of Physics and State Key Laboratory of Nuclear Physics and Technology, Peking University, Beijing 100871, China

E-mail: shakalee@pku.edu.cn, dxzhang@pku.edu.cn

ABSTRACT: We propose a mechanism for sufficient suppression of dimension-5 operators for proton decay in a supersymmetric $\mathrm{SO}(10)$ model. This mechanism is analogue to the double seesaw mechanism in studying neutrino masses. Only an intermediate VEV instead of an intermediate scale is required so that gauge coupling unification is maintained. The VEV is generated by introducing an anomalous U(1) symmetry whose breaking is at higher scale. The proton decay amplitudes are suppressed by this VEV over the GUT scale. We use $\mathbf{4 5 + 5 4}$ in breaking GUT symmetry. $\mathbf{1 2 0}$ is included so that fermion sector is fully realistic. Assuming a minimal fine-tuning in the Higgs doublet sector, $\tan \beta$ of order one is predicted.

Keywords: GUT, Neutrino Physics

ArXiv EPRINT: 1409.5233 


\section{Contents}

1 Introduction 1

2 General consideration on proton decay suppression 2

3 The model and SUSY preserving 3

4 Proton decay suppression $\quad 6$

5 The weak doublets $\quad 9$

$\begin{array}{lll}6 & \text { Summery and conclusions } & 10\end{array}$

\section{Introduction}

Grand Unified Theory (GUT) [1,2] is one of the most attractive candidates for the physics beyond the Standard Model (SM). The Supersymmetric (SUSY) GUT models based on $\mathrm{SO}(10)[3,4]$ are especially interesting for several reasons. Firstly, each generation of fermion superfields are unified in a single 16-plet spinor representation which contains the right-handed neutrino, so that sub-eV neutrino masses can be generated naturally by the seesaw mechanism [5-13]. Secondly, in the renormalizable versions of SUSY SO(10) models [14-16], R-parity is conserved automatically which eliminates the most dangerous dimension- 4 operators of proton decay.

The elegant running behaviors of the coupling constants in MSSM strongly suggest that the unification scale should be taken at $2 \times 10^{16} \mathrm{GeV}[17-22]$ which we call the GUT scale $M_{G}$. Not only the three coupling constants are unified at $M_{G}$, but also the masses of the gauge superfields $\mathrm{G}(\mathrm{SO}(10)) / \mathrm{G}(\mathrm{SM})$ are taken at the same scale. It has been recognized recently [23] that instead of an intermediate seesaw scale, in SUSY SO(10) models with several pairs of $\mathbf{1 2 6}+\overline{\mathbf{1 2 6}}$, only an intermediate vacuum expectation value (VEV) of the SM singlet in one $\overline{\mathbf{1 2 6}}$ is needed which couples with the matter superfields. Consequently, the spectra of this kind of models do not contain particles at intermediate scale so that gauge coupling unification is maintained, meanwhile the seesaw mechanism still works. This mechanism is further incorporated in models aiming at sufficiently suppressing proton decay [24], where the seesaw VEV is related to the VEV of an $\mathrm{SO}(10)$ singlet which breaks an extra global U(1) symmetry. Proton decay amplitudes are found to be suppressed in [24] by a factor $\frac{M_{I}}{M_{G}}$, where $M_{I} \sim 10^{14} \mathrm{GeV}$ is the seesaw VEV which is much smaller than the GUT scale $M_{G} \sim 10^{16} \mathrm{GeV}$. This suppression of proton decay is archived by the enhancements of the effective triplet masses through an inversely analogue to the mass texture in the seesaw mechanism, or a lever mechanism. 
In the present work we will extend the observation made by the previous study that the seesaw VEV might be related to the suppression of proton decay in other models. Instead of using 210 to break $\mathrm{SO}(10)$, we will use $\mathbf{4 5 + 5 4}$. The global U(1) will be replaced by an anomalous $\mathrm{U}(1)$ whose breaking is generated by an $\mathrm{SO}(10)$ singlet through the GreenShwarz mechanism $[25,26]$. The enhancements of the effective triplet masses responsible for proton decay are through an inversely analogue to the mass texture in the double-seesaw mechanism $[27,28]$. We will not improve on either the running behavior of the $\mathrm{SO}(10)$ gauge coupling above the GUT scale or on the minimal fine-tuning for the weak doublets which is implicitly assumed.

In the next section, we will give a simple overview on proton decay suppression. Then, we will propose in section 3 a renormalizable model and show its consistency with high energy supersymmetry. Proton decay suppression mechanism in this model is shown in section 4. The discussion on the weak doublets of the MSSM and the prediction of small $\tan \beta$ are followed in section 5 . We will summarize in section 6 .

\section{General consideration on proton decay suppression}

Consider a simplified model with two pairs of color triplets-anti-triplets, with only one pair of them couple with fermions. The mass term for the triplets can be written as $\left(\varphi_{\bar{T}}\right)_{i}(M)_{i j}\left(\varphi_{T}\right)_{j}$, where $i, j$ run from 1 to 2 . We need to rotate to the mass eigenstates in order to calculate proton decay amplitudes. Two $2 \times 2$ unitary matrices $U$ and $V$ are introduced as

$$
M_{i j}^{\prime}=U_{i k} M_{k l} V_{l j}^{\dagger}
$$

where $M^{\prime}=\operatorname{diag}\left(M_{1}, M_{2}\right)$. The mass eigenstates $\varphi_{\bar{T}}^{\prime} \mathrm{s}$ and $\varphi_{T}^{\prime} \mathrm{s}$ are

$$
\left(\varphi_{\bar{T}}^{\prime}\right)_{i}=\left(\varphi_{\bar{T}}\right)_{j} U_{j i}^{\dagger}, \quad\left(\varphi_{T}^{\prime}\right)_{i}=V_{i j}\left(\varphi_{T}\right)_{j}
$$

Then the dimension-5 operators mediated by the color triplet higgsinos are proportional to $[29]$

$$
\sum_{i} V_{1 i}^{\dagger} \frac{1}{M_{i i}^{\prime}} U_{i 1}=\left(V^{\dagger} \cdot M^{\prime-1} \cdot U\right)_{11}=\left[\left(U^{\dagger} \cdot M^{\prime} \cdot V\right)^{-1}\right]_{11}=\left(M^{-1}\right)_{11}
$$

The inverse of $\left(M^{-1}\right)_{11}$ is called the effective triplet mass which mimics the role of the color triplet higgsino in the simplest models with only one pair of color triplet-anti-triplet.

Eq. (2.3) is easy to be generalized to models with more pairs of color triplets-antitriplets. The proton decay amplitudes are proportional to sums of specific elements in the inverse of the triplet mass matrix. Algebraically, these matrix elements in the inverse mass matrix can be written as

$$
\left(M^{-1}\right)_{i j}=(-1)^{i+j} \frac{\operatorname{Det}\left(M_{j i}^{*}\right)}{\operatorname{Det}(M)},
$$

where $M_{i j}^{*}$ represents $M$ with the $i$ th row and the $j$ th column eliminated, whose determinant is called as the algebraic complement, and $i$ and $j$ are the labels of those color triplets-anti-triplets which can couple with the fermions. 
There are two possible ways to get small $\left(M^{-1}\right)_{i j}$ 's following (2.4). We can construct a mass matrix either with all small algebraic complements for the elements which couple with fermions, or with a large determinant of the entire mass matrix. In the previous work [24] the first approach is used where the color triplet mass matrix can be symbolically expressed as

$$
M_{T}=\left(\begin{array}{cc}
0 & M_{G} \\
M_{G} & M_{I}
\end{array}\right) .
$$

Here $M_{G}$ stands for a GUT scale mass while $M_{I}$ is the intermediate seesaw VEV. Only the up-left block couples with matter fields, so it is clear that $M_{11}^{*}=M_{I}$ is smaller than $M_{G}$.

In this work, we are trying to realize the second possibility. The mass matrix for the color triplets is written as

$$
M_{T}=\left(\begin{array}{ccc}
0 & M_{G} & 0 \\
M_{G} & 0 & M_{G} \\
0 & M_{G} & M_{P}
\end{array}\right) .
$$

Again, the matter fields couple with the up-left block only. Here $M_{P}$ represents a mass at a scale higher than the GUT scale, or at the Plank scale. Then $\operatorname{Det}\left(M_{T}\right) \sim M_{P} M_{G}^{2}$ is enhanced to give large effective triplet masses.

As the texture in (2.5) is analogue to the neutrino mass matrix in the seesaw mechanism, the present texture in (2.6) is analogue to the neutrino mass matrix in the doubleseesaw mechanism. The mass texture either in (2.5) or in (2.6) is sufficient to suppress proton decay.

\section{$3 \quad$ The model and SUSY preserving}

The particle content of the present model is as follows. First, it contains three generations of fermion fields which are embedded into three 16-plet $\left(\psi_{1,2,3}\right)$ superfields as usual. Second, $\mathbf{4 5}+\mathbf{5 4}(A, E)$ are introduced to break $\mathrm{SO}(10)$. Third, in order to give satisfied fermion masses and mixing [30], Higgs in $\mathbf{1 2 0}(D)$ is introduced, which is also needed to couple through $\mathbf{4 5}+\mathbf{5 4}$ with those in $\mathbf{1 0}(H)$ and in $\mathbf{1 2 6} / \overline{\mathbf{1 2 6}}(\Delta / \bar{\Delta})$. Forth, the $\mathbf{4 5}$ is further copied $\left(A^{\prime}\right)$ to generate a small VEV for the seesaw mechanism, and to generate the structure (2.6) for suppression of proton decay. Three sets of Higgs are needed with the first two sets contain $H+\Delta / \bar{\Delta}+D$ while the third set contains $\Delta / \bar{\Delta}$. An extra U(1) symmetry, whose breaking is realized by the $\mathrm{SO}(10)$ singlets $S_{1}$ and $S_{2}$, is introduced to distinguish these Higgs. All the fields and their $\mathrm{U}(1)$ charges are listed in table 1. Note that the different $\mathrm{U}(1)$ charges of the first set of Higgs $\left(H_{1} \ldots\right)$ and the third set of Higgs $\left(\Delta_{3} \ldots\right)$ also require different fields $\left(A+E\right.$ and $\left.A^{\prime}\right)$ to couple the first two and the last two sets of Higgs.

Only $H_{1}, D_{1}, \bar{\Delta}_{1}$ couple with matter fields due to the U(1) charges. The Yukawa sector is given as

$$
W_{Y}=Y_{10}^{i j} \psi_{i} \psi_{j} H_{1}+Y_{120}^{i j} \psi_{i} \psi_{j} D_{1}+Y_{126}^{i j} \psi_{i} \psi_{j} \bar{\Delta}_{1},
$$

which is general enough to fit all fermion masses and mixing [15, 31-39]. 


\begin{tabular}{|c|c|c|c|c|c|c|c|c|}
\hline & $\psi_{i}$ & $H_{1}, D_{1}, \Delta_{1} / \bar{\Delta}_{1}$ & $H_{2}, D_{2}, \Delta_{2} / \bar{\Delta}_{2}$ & $\Delta_{3} / \bar{\Delta}_{3}$ & $A, E$ & $A^{\prime}$ & $S_{1}$ & $S_{2}$ \\
\hline $\mathrm{U}(1)$ charge & $-\frac{1}{2}$ & 1 & -1 & $\frac{1}{2}$ & 0 & $\frac{1}{2}$ & -1 & $-\frac{1}{2}$ \\
\hline
\end{tabular}

Table 1. SO(10) multiplets and their U(1) charges.

The general renormalizable Higgs superpotential is given by

$$
\begin{aligned}
W= & m_{H} H_{1} H_{2}+m_{\Delta_{12}} \bar{\Delta}_{1} \Delta_{2}+m_{\Delta_{21}} \bar{\Delta}_{2} \Delta_{1}+m_{D} D_{1} D_{2}+\frac{1}{2} m_{A} A^{2}+\frac{1}{2} m_{E} E^{2} \\
& +H_{1} H_{2}\left(\lambda_{1} A+\lambda_{2} E\right)-i A\left(\lambda_{3} \bar{\Delta}_{1} \Delta_{2}+\lambda_{4} \bar{\Delta}_{2} \Delta_{1}\right)+E\left(\lambda_{5} \Delta_{1} \Delta_{2}+\lambda_{6} \bar{\Delta}_{1} \bar{\Delta}_{2}\right) \\
& +D_{1} A\left(\lambda_{7} H_{2}+\lambda_{8} \Delta_{2}+\lambda_{9} \bar{\Delta}_{2}\right)+D_{2} A\left(\lambda_{10} H_{1}+\lambda_{11} \Delta_{1}+\lambda_{12} \bar{\Delta}_{1}\right) \\
& +D_{1} D_{2}\left(\lambda_{13} A+\lambda_{14} E\right)+\lambda_{15} E^{3}+\lambda_{16} A E^{2}-i A^{\prime}\left(\alpha_{1} \bar{\Delta}_{2} \Delta_{3}+\alpha_{2} \bar{\Delta}_{3} \Delta_{2}\right) \\
& +D_{2} A^{\prime}\left(\alpha_{3} \Delta_{3}+\alpha_{4} \bar{\Delta}_{3}\right)+\frac{1}{2} S_{1}\left(2 \beta_{1} \bar{\Delta}_{3} \Delta_{3}+\beta_{2} A^{\prime 2}\right)+\beta_{3} S_{2} A A^{\prime} .
\end{aligned}
$$

Labeled by the representations under the $\mathrm{SU}(4)_{C} \times \mathrm{SU}(2)_{L} \times \mathrm{SU}(2)_{R}$ subgroup of $\mathrm{SO}(10)$, the following components get VEVs responsible for the $\mathrm{SO}(10)$ symmetry breaking

$$
\begin{aligned}
A_{1}^{(\prime)} & =\left\langle A^{(\prime)}(1,1,3)\right\rangle, A_{2}^{(\prime)}=\left\langle A^{(\prime)}(15,1,1)\right\rangle, E=\langle E(1,1,1)\rangle ; \\
v_{(1,2,3)} & =\left\langle\Delta_{(1,2,3)}(\overline{10}, 1,3)\right\rangle, \bar{v}_{(1,2,3)}=\left\langle\bar{\Delta}_{(1,2,3)}(10,1,3)\right\rangle .
\end{aligned}
$$

Inserting these VEVs into (3.2), we get

$$
\begin{aligned}
\langle W\rangle= & m_{\Delta 12} \bar{v}_{1} v_{2}+m_{\Delta 21} \bar{v}_{2} v_{1}+\frac{1}{2} m_{A}\left(A_{1}^{2}+A_{2}^{2}\right)+\frac{1}{2} m_{E} E^{2}+A_{0}\left(\lambda_{3} \bar{v}_{1} v_{2}+\lambda_{4} \bar{v}_{2} v_{1}\right) \\
& +\frac{\lambda_{15}}{2 \sqrt{15}} E^{3}+\lambda_{16} E\left(\frac{\sqrt{3}}{2 \sqrt{5}} A_{1}^{2}-\frac{1}{\sqrt{15}} A_{2}^{2}\right)+A_{0}^{\prime}\left(\alpha_{1} \bar{v}_{2} v_{3}+\alpha_{2} \bar{v}_{3} v_{2}\right) \\
& +\frac{1}{2} S_{1}\left(2 \beta_{1} \bar{v}_{3} v_{3}+\beta_{2} A_{1}^{\prime 2}+\beta_{2} A_{2}^{\prime 2}\right)+S_{2}\left(\beta_{3} A_{1} A_{1}^{\prime}+\beta_{3} A_{2} A_{2}^{\prime}\right)
\end{aligned}
$$

where we have defined

$$
A_{0} \equiv\left(-\frac{1}{5} A_{1}-\frac{3}{5 \sqrt{6}} A_{2}\right), \quad A_{0}^{\prime} \equiv\left(-\frac{1}{5} A_{1}^{\prime}-\frac{3}{5 \sqrt{6}} A_{2}^{\prime}\right),
$$

for later convenience.

To preserve SUSY at high energy, the F- and D-flatness conditions are required. The D-flatness condition requires

$$
\left|v_{1}\right|^{2}+\left|v_{2}\right|^{2}+\left|v_{3}\right|^{2}=\left|\bar{v}_{1}\right|^{2}+\left|\bar{v}_{2}\right|^{2}+\left|\bar{v}_{3}\right|^{2},
$$

which constrains only the sum of $|v|^{2}$ s and $|\bar{v}|^{2}$ s, so that an intermediate valued VEV of $\bar{v}_{1}$ can be generated without breaking gauge coupling unification, if both sides in (3.6) are of the order $M_{G}^{2}$.

The F-flatness conditions

$$
\left\{\frac{\partial}{\partial v_{1}}, \frac{\partial}{\partial v_{2}}, \frac{\partial}{\partial v_{3}}, \frac{\partial}{\partial \bar{v}_{1}}, \frac{\partial}{\partial \bar{v}_{2}}, \frac{\partial}{\partial \bar{v}_{3}}, \frac{\partial}{\partial A_{1}^{\prime}}, \frac{\partial}{\partial A_{2}^{\prime}}, \frac{\partial}{\partial S_{1}}, \frac{\partial}{\partial S_{2}}, \frac{\partial}{\partial A_{1}}, \frac{\partial}{\partial A_{2}}, \frac{\partial}{\partial E}\right\}\langle W\rangle=0,
$$


are explicitly

$$
\begin{aligned}
& 0=M_{21} \bar{v}_{2} \\
& 0=M_{12} \bar{v}_{1}+\alpha_{2} A_{0}^{\prime} \bar{v}_{3}, \\
& 0=\alpha_{1} A_{0}^{\prime} \bar{v}_{2}+\beta_{1} S_{1} \bar{v}_{3}, \\
& 0=M_{12} v_{2} \\
& 0=M_{21} v_{1}+\alpha_{1} A_{0}^{\prime} v_{3}, \\
& 0=\alpha_{2} A_{0}^{\prime} v_{2}+\beta_{1} S_{1} v_{3}, \\
& 0=\beta_{2} S_{1} A_{1}^{\prime}+\beta_{3} S_{2} A_{1}-\frac{1}{5}\left(\alpha_{1} \bar{v}_{2} v_{3}+\alpha_{2} \bar{v}_{3} v_{2}\right), \\
& 0=\beta_{2} S_{1} A_{2}^{\prime}+\beta_{3} S_{2} A_{2}-\frac{3}{5 \sqrt{6}}\left(\alpha_{1} \bar{v}_{2} v_{3}+\alpha_{2} \bar{v}_{3} v_{2}\right), \\
& 0=\beta_{1} \bar{v}_{3} v_{3}+\beta_{2} A_{1}^{\prime 2}+\beta_{2} A_{2}^{\prime 2} \\
& 0=\beta_{3} A_{1} A_{1}^{\prime}+\beta_{3} A_{2} A_{2}^{\prime} \\
& 0=m_{A} A_{1}-\frac{1}{5}\left(\lambda_{3} \bar{v}_{1} v_{2}+\lambda_{4} \bar{v}_{2} v_{1}\right)+\frac{\sqrt{3}}{\sqrt{5}} \lambda_{16} E A_{1}+\beta_{3} A_{1}^{\prime} S_{2}, \\
& 0=m_{A} A_{2}-\frac{3}{5 \sqrt{6}}\left(\lambda_{3} \bar{v}_{1} v_{2}+\lambda_{4} \bar{v}_{2} v_{1}\right)-\frac{2}{\sqrt{15}} \lambda_{16} E A_{2}+\beta_{3} A_{2}^{\prime} S_{2}, \\
& 0=m_{E} E+\frac{3}{2 \sqrt{15}} \lambda_{15} E^{2}+\lambda_{16}\left(\frac{\sqrt{3}}{2 \sqrt{5}} A_{1}^{2}-\frac{1}{\sqrt{15}} A_{2}^{2}\right),
\end{aligned}
$$

where

$$
M_{12} \equiv m_{\Delta 12}+\lambda_{3} A_{0}, \quad M_{21} \equiv m_{\Delta 21}+\lambda_{4} A_{0} .
$$

From (3.6) to (3.19), there are 13 variables and 14 equations in total, but only 12 of the equations are independent. One of the $\mathrm{VEVs}, S_{1}$ for example, can be assigned to any scale.

First, (3.7)-(3.9) are linear equations about the $\bar{v}$ s, which can be rewritten as

$$
\left(\bar{v}_{1}, \bar{v}_{2}, \bar{v}_{3}\right)\left(\begin{array}{ccc}
0 & M_{12} & 0 \\
M_{21} & 0 & \alpha_{1} A_{0}^{\prime} \\
0 & \alpha_{2} A_{0}^{\prime} & \beta_{1} S_{1}
\end{array}\right)=0 .
$$

Similarly, equations (3.10)-(3.12) can be rewritten as

$$
\left(\begin{array}{ccc}
0 & M_{12} & 0 \\
M_{21} & 0 & \alpha_{1} A_{0}^{\prime} \\
0 & \alpha_{2} A_{0}^{\prime} & \beta_{1} S_{1}
\end{array}\right)\left(\begin{array}{l}
v_{1} \\
v_{2} \\
v_{3}
\end{array}\right)=0 .
$$

Both (3.21) and (3.22) require

$$
\beta_{1} S_{1} M_{12} M_{21}=0,
$$

which corresponds to three different possibilities as following. If $S_{1}=0$, a lot of particles cannot get masses through couplings with $S_{1}$. Thus this case is simply excluded. If $M_{12}=0$ 
is taken, then from (3.21)-(3.22) it follows that $\bar{v}_{1}=M_{G}$ and $\bar{v}_{2}=\bar{v}_{3}=0$ which gives too small neutrino masses and is thus excluded as well. We thus have the last possibility,

$$
M_{21}=0,
$$

which gives

$$
v_{1} \sim M_{G}, v_{2}=v_{3}=0, \quad \frac{\bar{v}_{1}}{\bar{v}_{3}}=-\frac{\alpha_{2} A_{0}^{\prime}}{M_{12}}, \frac{\bar{v}_{2}}{\bar{v}_{3}}=-\frac{\beta_{1} S_{1}}{\alpha_{1} A_{0}^{\prime}},
$$

following (3.21) and (3.22). Then, $\bar{v}_{1,2,3}$ are expressed by $v_{1}$ through (3.6).

Furthermore, substituting $v_{2}=v_{3}=0$ into (3.13)-(3.14), $A_{1}^{\prime}$ and $A_{2}^{\prime}$ can be expressed by $A_{1}$ and $A_{2}$, respectively, and (3.16) is now identical to (3.15). Equation $M_{21}=0$ in (3.24) itself gives the dependence of $A_{2}$ on $A_{1}$ through (3.20). Taking $S_{1}$ as free, the remaining variables are now $A_{1}, E, S_{2}, v_{1}$ with equations (3.15), (3.17), (3.18) and (3.19) left. Given the parameters in (3.2), all the VEVs are now determined. Numerically, $A_{1}$, $A_{2}$ and $E$ are taken as GUT scale VEVs in order to break SO(10) down to MSSM.

According to the analysis in [40], the extra $\mathrm{U}(1)$ symmetry is naturally related to string theory, and it is appropriate to take the VEV of breaking this $\mathrm{U}(1)$ at

$$
S_{1} \sim 10^{17} \mathrm{GeV} \sim 10 M_{G} .
$$

After inserting (3.13)-(3.14) into (3.17)-(3.18), the last terms will change into

$$
\beta_{3} A_{1}^{\prime} S_{2} \rightarrow-\frac{\beta_{3}^{2} S_{2}^{2}}{\beta_{2} S_{1}} A_{1}, \quad \beta_{3} A_{2}^{\prime} S_{2} \rightarrow-\frac{\beta_{3}^{2} S_{2}^{2}}{\beta_{2} S_{1}} A_{2} .
$$

They are naturally at the same scale as other terms, i.e. $M_{G}^{2}$, which indicates that

$$
S_{2} \sim \frac{1}{\sqrt{10}} S_{1} \sim \sqrt{10} M_{G}, \quad A_{1}^{\prime} \sim A_{2}^{\prime} \sim \frac{1}{\sqrt{10}} M_{G} .
$$

Thus we get from (3.25)

$$
\left(v_{1}, v_{2}, v_{3}\right)=\left(O\left(M_{G}\right), 0,0\right) \quad\left(\bar{v}_{1}, \bar{v}_{2}, \bar{v}_{3}\right)=O\left(10^{-2} M_{G}, M_{G}, 10^{-\frac{3}{2}} M_{G}\right) .
$$

Now that all the constrains on SUSY preserving have been satisfied, all the VEVs can be determined and all their scales are known. The seesaw VEV $\bar{v}_{1} \sim M_{I}$ is naturally generated at $10^{-2} M_{G}$, which differs from [24] where it introduced a VEV of an $\mathrm{SO}(10)$ singlet which broke a global $\mathrm{U}(1)$ symmetry.

Consequence of the large masses of the third set of Higgs and $A^{\prime}$ given by the VEV $S_{1} \sim 10 M_{G}$ is that these Higgs are to be integrated out above the GUT scale $M_{G}$, so that they do not affect the running behaviors of gauge couplings of MSSM.

\section{Proton decay suppression}

To demonstrate the effectiveness of the present model on solving the proton decay problem, we need to write down the color triplet mass matrix. The color triplets are ordered as

$$
\varphi_{T}=\left(H_{1 T}, D_{1 T}, D_{1 T}^{\prime}, \Delta_{1 T}, \bar{\Delta}_{1 T}, \bar{\Delta}_{1 T}^{\prime} ; H_{2 T}, D_{2 T}, D_{2 T}^{\prime}, \Delta_{2 T}, \bar{\Delta}_{2 T}, \bar{\Delta}_{2 T}^{\prime} ; \Delta_{3 T}, \bar{\Delta}_{3 T}, \bar{\Delta}_{3 T}^{\prime}\right) \text {, }
$$


while the color anti-triplets are

$$
\varphi_{\bar{T}}=\left(H_{1 \bar{T}}, D_{1 \bar{T}}, D_{1 \bar{T}}^{\prime}, \bar{\Delta}_{1 \bar{T}}, \Delta_{1 \bar{T}}, \Delta_{1 \bar{T}}^{\prime} ; H_{2 \bar{T}}, D_{2 \bar{T}}, D_{2 \bar{T}}^{\prime}, \bar{\Delta}_{2 \bar{T}}, \Delta_{2 \bar{T}}, \Delta_{2 \bar{T}}^{\prime} ; \bar{\Delta}_{3 \bar{T}}, \Delta_{3 \bar{T}}, \Delta_{3 \bar{T}}^{\prime}\right) .
$$

The mass term of the Higgs color triplets is given by $\left(\varphi_{\bar{T}}\right)_{a}\left(M_{T}\right)_{a b}\left(\varphi_{T}\right)_{b}$, with the $15 \times 15$ matrix $M_{T}$ written as

$$
M_{T}=\left(\begin{array}{ccc}
0_{(6 \times 6)} & B_{12(6 \times 6)} & 0_{(6 \times 3)} \\
B_{21(6 \times 6)} & 0_{(6 \times 6)} & B_{23(6 \times 3)} \\
0_{(3 \times 6)} & B_{32(3 \times 6)} & B_{33(3 \times 3)}
\end{array}\right),
$$

where

$$
\begin{aligned}
& B_{12}=\left(\begin{array}{cccccc}
B_{H 12} & -\frac{i \lambda_{10}}{\sqrt{3}} A_{1} & -\frac{i \sqrt{2} \lambda_{10}}{3} A_{2} & 0 & 0 & 0 \\
\frac{i \lambda_{7}}{\sqrt{3}} A_{1} & B_{D 12} & 0 & -\frac{i \lambda_{8}}{2 \sqrt{5}} A_{1} & -\frac{i \lambda_{9}}{2 \sqrt{5}} A_{1} & -\frac{i \lambda_{9}}{\sqrt{15}} A_{2} \\
-\frac{i \sqrt{2} \lambda_{7}}{3} A_{2} & 0 & B_{D 12}^{\prime} & \frac{i \lambda_{8}}{\sqrt{30}} A_{2} & -\frac{i \lambda_{9}}{\sqrt{30}} A_{2} & -\frac{i \lambda_{9}}{\sqrt{10}} A_{1} \\
0 & \frac{i \lambda_{12}}{2 \sqrt{5}} A_{1} & -\frac{i \lambda_{12}}{\sqrt{30}} A_{2} & m_{\Delta 12}+\frac{\lambda_{3}}{5 \sqrt{6}} A_{2} & \frac{2 \lambda_{6}}{\sqrt{15}} E & 0 \\
0 & \frac{i \lambda_{11}}{2 \sqrt{5}} A_{1} & \frac{i \lambda_{11}}{\sqrt{30}} A_{2} & \frac{2 \lambda_{5}}{\sqrt{15}} E & m_{\Delta 21}-\frac{\lambda_{4}}{5 \sqrt{6}} A_{2} & 0 \\
0 & \frac{i \lambda_{11}}{\sqrt{15}} A_{2} & \frac{i \lambda_{11}}{\sqrt{10}} A_{1} & 0 & 0 & m_{\Delta 21}-\frac{\lambda_{4}}{5 \sqrt{6}} A_{2}
\end{array}\right), \\
& B_{21}=\left(\begin{array}{cccccc}
B_{H 21} & -\frac{i \lambda_{7}}{\sqrt{3}} A_{1} & -\frac{i \sqrt{2} \lambda_{7}}{3} A_{2} & 0 & 0 & 0 \\
\frac{i \lambda_{10}}{\sqrt{3}} A_{1} & B_{D 21} & 0 & -\frac{i \lambda_{11}}{2 \sqrt{5}} A_{1} & -\frac{i \lambda_{12}}{2 \sqrt{5}} A_{1} & -\frac{i \lambda_{12}}{\sqrt{15}} A_{2} \\
-\frac{i \sqrt{2} \lambda_{10}}{3} A_{2} & 0 & B_{D 21}^{\prime} & \frac{i \lambda_{11}}{\sqrt{30}} A_{2} & -\frac{i \lambda_{12}}{\sqrt{30}} A_{2} & -\frac{i \lambda_{12}}{\sqrt{10}} A_{1} \\
0 & \frac{i \lambda_{9}}{2 \sqrt{5}} A_{1} & -\frac{i \lambda_{9}}{\sqrt{30}} A_{2} & m_{\Delta 21}+\frac{\lambda_{4}}{5 \sqrt{6}} A_{2} & \frac{2 \lambda_{6}}{\sqrt{15}} E & 0 \\
0 & \frac{i \lambda_{8}}{2 \sqrt{5}} A_{1} & \frac{i \lambda_{8}}{\sqrt{30}} A_{2} & \frac{2 \lambda_{5}}{\sqrt{15}} E & m_{\Delta 12}-\frac{\lambda_{3}}{5 \sqrt{6}} A_{2} & 0 \\
0 & \frac{i \lambda_{8}}{\sqrt{15}} A_{2} & \frac{i \lambda_{8}}{\sqrt{10}} A_{1} & 0 & 0 & m_{\Delta 12}-\frac{\lambda_{3}}{5 \sqrt{6}} A_{2}
\end{array}\right), \\
& B_{23}=\left(\begin{array}{ccc}
0 & 0 & 0 \\
-\frac{i \alpha_{3}}{2 \sqrt{5}} A_{1}^{\prime} & -\frac{i \alpha_{4}}{2 \sqrt{5}} A_{1}^{\prime} & -\frac{i \alpha_{4}}{\sqrt{15}} A_{2}^{\prime} \\
\frac{i \alpha_{3}}{\sqrt{30}} A_{2}^{\prime} & -\frac{i \alpha_{4}}{\sqrt{30}} A_{2}^{\prime} & -\frac{i \alpha_{4}}{\sqrt{10}} A_{1}^{\prime} \\
\frac{\alpha_{1}}{5 \sqrt{6}} A_{2}^{\prime} & 0 & 0 \\
0 & -\frac{\alpha_{2}}{5 \sqrt{6}} A_{2}^{\prime} & 0 \\
0 & 0 & -\frac{\alpha_{2}}{5 \sqrt{6}} A_{2}^{\prime}
\end{array}\right),
\end{aligned}
$$

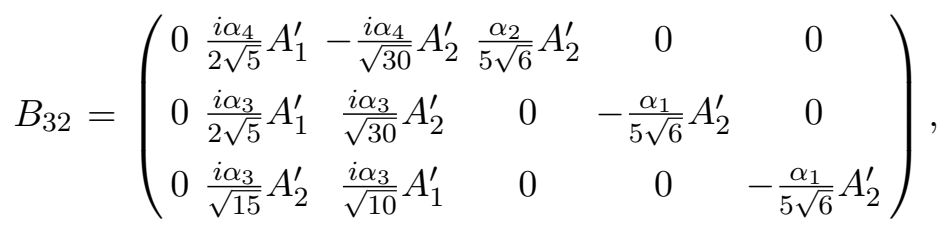


and

$$
B_{33}=\left(\begin{array}{ccc}
\beta_{1} S_{1} & 0 & 0 \\
0 & \beta_{1} S_{1} & 0 \\
0 & 0 & \beta_{1} S_{1}
\end{array}\right)
$$

Here

$$
\begin{aligned}
B_{H 12} & \equiv m_{H}+\frac{i \lambda_{1}}{\sqrt{6}} A_{2}+\frac{2 \lambda_{2}}{\sqrt{15}} E, & B_{H 21} & \equiv m_{H}-\frac{i \lambda_{1}}{\sqrt{6}} A_{2}+\frac{2 \lambda_{2}}{\sqrt{15}} E \\
B_{D 12} & \equiv m_{D}+\frac{i \lambda_{13}}{3 \sqrt{6}} A_{2}+\frac{4 \lambda_{14}}{3 \sqrt{15}} E, & B_{D 21} & \equiv m_{D}-\frac{i \lambda_{13}}{3 \sqrt{6}} A_{2}+\frac{4 \lambda_{14}}{3 \sqrt{15}} E \\
B_{D 12}^{\prime} & \equiv m_{D}+\frac{i \lambda_{13}}{3 \sqrt{6}} A_{2}-\frac{2 \lambda_{14}}{\sqrt{15}} E, & B_{D 21}^{\prime} & \equiv m_{D}-\frac{i \lambda_{13}}{3 \sqrt{6}} A_{2}-\frac{2 \lambda_{14}}{\sqrt{15}} E
\end{aligned}
$$

The mass matrix can be also expressed symbolically as

$$
M_{T}=\left(\begin{array}{ccc}
0_{(6 \times 6)} & M_{G(6 \times 6)} & 0_{(6 \times 3)} \\
M_{G(6 \times 6)} & 0_{(6 \times 6)} & \frac{1}{\sqrt{10}} M_{G(6 \times 3)} \\
0_{(3 \times 6)} & \frac{1}{\sqrt{10}} M_{G(3 \times 6)} & 10 M_{G(3 \times 3)}
\end{array}\right) .
$$

Note that the texture in (4.9), constrained by the F- and D-flatness conditions, differs slightly from that in (2.6). However, as will be seen in the rest of this section, the mechanism of suppressing proton decay following (2.6) will not change.

In SUSY GUTs, the dominant channels inducing proton decay are through the dimension-5 operators $[41,42]$

$$
-W_{5}=C_{L}^{i j k l} \frac{1}{2} q_{i} q_{j} q_{k} l_{l}+C_{R}^{i j k l} u_{i}^{c} d_{j}^{c} u_{k}^{c} e_{l}^{c}
$$

which are called the $L L L L$ and $R R R R$ operators, respectively, obtained by integrating out the color triplet and anti-triplet Higgs superfields in the interactions in (3.1). Both $C_{L}^{i j k l}$ and $C_{R}^{i j k l}$ are inversely proportional to the effective mass of the colored Higgsino. Since only $B_{11}$ part couples with fermions, we can get the effective mass by integrating out the uncoupled parts. From (4.9), such a mass matrix is similar to the mass matrix in the double seesaw models for neutrino masses [27, 28] which is used to generate the small neutrino masses. In the present model, the effective masses are large instead of small because $B_{23} \sim B_{32} \ll B_{12} \sim B_{21} \ll B_{33}$. Similarly, this proton decay suppression mechanism requires two steps of integrations. Since $S_{1}$ is ten times of the GUT scale, the $B_{33}$ part can be integrate out first. Then the mass matrix becomes

$$
M_{T}=\left(\begin{array}{cc}
0_{(6 \times 6)} & M_{G(6 \times 6)} \\
M_{G(6 \times 6)} & M_{I(6 \times 6)}
\end{array}\right),
$$

where

$$
M_{I(6 \times 6)}=-B_{23} \cdot B_{33}^{-1} \cdot B_{32},
$$

is a matrix with all elements of the order $10^{-2} M_{G}$. Then after the second step,

$$
M_{\mathrm{eff}}=-B_{21} \cdot M_{I(6 \times 6)}^{-1} \cdot B_{12} \sim \frac{M_{G}^{2}}{M_{I}} \sim 2 \times 10^{18} \mathrm{GeV},
$$


which is of the order of the Plank scale, a hundred times heavier than those color triplet Higgs masses in SUSY GUT models. The proton decay rates will be suppressed by a factor of $10^{-4}$, which is small enough surviving all the current experimental limits. One may have found that $M_{I(6 \times 6)}$ is a rank 3 matrix and is thus not reversible. This is because that we have not introduced the third $\mathbf{1 0}+\mathbf{1 2 0}$-plet $H_{3}+D_{3}$ for simplicity. But, as was discussed in $[23,24]$, each rank contributes one eigenvalue in the effective masses. The diagonal form of the effective mass matrix is

$$
M_{\mathrm{eff}}=\operatorname{diag} O\left(\frac{M_{G}^{2}}{M_{I}}, \frac{M_{G}^{2}}{M_{I}}, \frac{M_{G}^{2}}{M_{I}}, \infty, \infty, \infty\right) .
$$

Note that it is the lightest eigenvalues that dominates the proton decay rates, while the three infinitely heavy masses do not contribute.

The suppression can be better understood if we write down the dimension- 5 operators explicitly. The coefficients $C_{L}$ s at the GUT scale $M_{G}$ are [43]

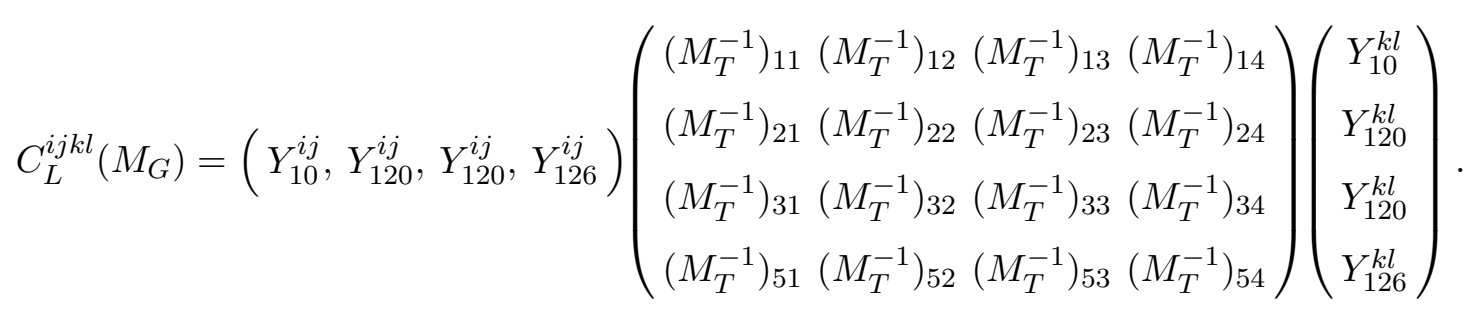

Here the Yukawa couplings are strongly constrained by fitting the fermion masses and mixing [33, 37-39]. The elements of $M_{T}^{-1}$ are of the order $\frac{1}{M_{G}}$ in usual SUSY GUT models, but in our model,

$$
M_{T}^{-1}=\frac{1}{M_{G}}\left(\begin{array}{ccc}
10_{(6 \times 6)}^{-2} & 1_{(6 \times 6)} & 10_{(6 \times 3)}^{-\frac{3}{2}} \\
1_{(6 \times 6)} & 0_{(6 \times 6)} & 0_{(6 \times 3)} \\
10_{(3 \times 6)}^{-\frac{3}{2}} & 0_{(3 \times 6)} & \frac{1}{10}(3 \times 3)
\end{array}\right) .
$$

We can see clearly that the elements contributing to dimension- 5 operators, i.e. elements in the up-left most block, are of the order $\frac{10^{-2}}{M_{G}}$. This is the same conclusion drawn in (4.13). This conclusion applies for both the LLLL and RRRR operators.

\section{The weak doublets}

Like in [24], the doublet-triplet splitting (DTS) problem requires a minimal fine-tuning, and similar results can be reached. The up-type doublets are ordered as

$$
\varphi_{u}=\left(H_{1 u}, D_{1 u}, D_{1 u}^{\prime}, \Delta_{1 u}, \bar{\Delta}_{1 u} ; H_{2 u}, D_{2 u}, D_{2 u}^{\prime}, \Delta_{2 u}, \bar{\Delta}_{2 u} ; \Delta_{3 u}, \bar{\Delta}_{3 u}\right),
$$

while down-type doublets are

$$
\varphi_{d}=\left(H_{1 d}, D_{1 d}, D_{1 d}^{\prime}, \bar{\Delta}_{1 d}, \Delta_{1 d} ; H_{2 d}, D_{2 d}, D_{2 d}^{\prime}, \bar{\Delta}_{2 d}, \Delta_{2 d} ; \bar{\Delta}_{3 d}, \Delta_{3 d}\right) .
$$


The mass terms of the weak doublets are given by $\left(\varphi_{d}\right)_{a}\left(M_{D}\right)_{a b}\left(\varphi_{u}\right)_{b}$, with the $12 \times 12$ matrix $M_{D}$ written as

$$
M_{D}=\left(\begin{array}{ccc}
0_{(5 \times 5)} & A_{12(5 \times 5)} & 0_{(5 \times 2)} \\
A_{21(5 \times 5)} & 0_{(5 \times 5)} & A_{23(5 \times 2)} \\
0_{(2 \times 5)} & A_{32(2 \times 5)} & A_{33(2 \times 2)}
\end{array}\right)=\left(\begin{array}{ccc}
0_{(5 \times 5)} & M_{G(5 \times 5)} & 0_{(5 \times 2)} \\
M_{G(5 \times 5)} & 0_{(5 \times 5)} & \frac{1}{\sqrt{10}} M_{G(5 \times 2)} \\
0_{(2 \times 5)} & \frac{1}{\sqrt{10}} M_{G(2 \times 5)} & 10 M_{G(2 \times 2)}
\end{array}\right) .
$$

For general parameters, there is no massless doublet. The DTS, which requires a zero determinant of $M_{D}$, can be obtained if

$$
\operatorname{Det}\left(M_{D}\right)=\operatorname{Det}\left(A_{12}\right) * \operatorname{Det}\left(A_{21}\right) * \operatorname{Det}\left(A_{33}\right)=0 .
$$

$\operatorname{Det}\left(A_{33}\right)$ is obviously nonzero, leaving us two choices. $\operatorname{Det}\left(A_{12}\right)=0$ is not acceptable because the large top quark mass would not be generated for perturbative Yukawa couplings. If we chose $\operatorname{Det}\left(A_{21}\right)=0$, we will further get the massless doublet can be expressed as

$$
H_{u}=\sum_{i=1}^{13} \alpha_{u}^{i *} \varphi_{u}^{i}, \quad H_{d}=\sum_{i=1}^{13} \alpha_{d}^{i *} \varphi_{d}^{i}
$$

and the components are, up to a normalization factor,

$$
\begin{aligned}
& \alpha_{u}^{*}=O(\underbrace{1, \ldots, 1}_{\text {five }} ; \underbrace{0, \ldots, 0}_{\text {seven }}), \\
& \alpha_{d}^{*}=O(\underbrace{10^{-2}, \ldots, 10^{-2}}_{\text {five }} ; \underbrace{1, \ldots, 1}_{\text {five }} ; \underbrace{10^{-\frac{3}{2}}, \ldots, 10^{-\frac{3}{2}}}_{\text {two }}) .
\end{aligned}
$$

The large ratio of $\frac{\alpha_{u}^{i}}{\alpha_{d}^{i}}(i \leq 5)$ is consistent with the ratio of $\frac{m_{t}}{m_{b}} \sim 100$ at high energy [33, 3739]. It also gives the constrain on $\tan \beta$

$$
\tan \beta=\frac{v_{u}}{v_{d}} \approx \frac{m_{t}}{m_{b}} 10^{-2} \sim O(1) .
$$

Equation (5.7) suggests that a small $\tan \beta$ is favored in the present model, which is also the same conclusion drawn in [24].

\section{Summery and conclusions}

As in [24], we do not perform explicitly the fine-tuning in the weak doublets which takes only one free parameter in the superpotential. As was pointed out in [30], threshold effects can be big in the minimal SUSY SU(5) theory, and can be even bigger due to the more super-heavy particles in $\mathrm{SO}(10)$ models. In this work, we have focused mainly on the new method of proton decay suppression and this method does not require explicit threshold effect calculations. The other reason is that there are more than enough free parameters in the superpotential that can be adjusted in calculating the threshold effects to fulfill the gauge coupling unification. 
In the present work we have presented a renormalizable SUSY SO(10) model with sufficient suppression of proton decay. Similar to [24], gauge coupling unification is maintained due to the absence of intermediate scales, and the seesaw VEV, proton decay and $\tan \beta$ are found to be all related, Thus the main conclusions are quite general in a class of models which follow the mechanisms of suppressing proton decay through constructing seesaw-like textures in the color triplet mass matrices. Different from the previous study, we use $\mathbf{4 5 + 5 4}$ instead of $\mathbf{2 1 0}$ to break $\mathrm{SO}(10)$. Instead of a global U(1) used in [24], we use an anomalous U(1) to generate the seesaw VEV through Green-Schwarz mechanism. We have also included 120-plet Higgs to couple with fermions so that the model is highly realistic. We have, however, two main problems untouched. The first is the DTS problem which we simply use an assumed fine-tuning in the weak doublets. The second is the perturbative difficulty for the gauge coupling above the GUT scale which is also common to all realistic SUSY GUT models.

We can compare our work with $[44,45]$ where Higgs in $\mathbf{1 0}+\mathbf{1 2 0}+\mathbf{1 2 6} / \overline{\mathbf{1 2 6}}$ are used to fit fermion masses and mixing while $\mathbf{2 1 0}$ is used to break $\mathrm{SO}(10)$. Proton decay suppression is carried out by raising the GUT scale up to the Planck scale or even higher so that the color-triplet Higgs masses are also enhanced accordingly, otherwise proton lifetime is around $10^{28} \mathrm{yr}$ only. This picture conflicts with the most important results supporting SUSY GUT which suggest the GUT scale to be $M_{G} \sim 2 \times 10^{16} \mathrm{GeV}$ [19-22]. In our work, the suppression of proton decay is realized by enhancement of the effective triplet masses and the unification scale remains at $M_{G}$.

Open Access. This article is distributed under the terms of the Creative Commons Attribution License (CC-BY 4.0), which permits any use, distribution and reproduction in any medium, provided the original author(s) and source are credited.

\section{References}

[1] H. Georgi and S.L. Glashow, Unity of All Elementary Particle Forces, Phys. Rev. Lett. 32 (1974) 438 [INSPIRE].

[2] J.C. Pati and A. Salam, Lepton number as the fourth "color", Phys. Rev. D 10 (1974) 275 [Erratum ibid. D 11 (1975) 703] [INSPIRE].

[3] T.E. Clark, T.-K. Kuo and N. Nakagawa, An $\mathrm{SO}(10)$ supersymmetric grand unified theory, Phys. Lett. B 115 (1982) 26 [INSPIRE].

[4] C.S. Aulakh and R.N. Mohapatra, Implications of Supersymmetric $\mathrm{SO}(10)$ Grand Unification, Phys. Rev. D 28 (1983) 217 [InSPIRE].

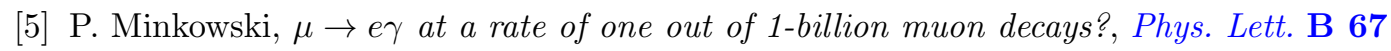
(1977) 421 [INSPIRE].

[6] T. Yanagida, Horizontal gauge symmetry and masses of neutrinos, in Proceedings of the Workshop on the Unified Theory and Baryon Number in the Universe, (1979), pg. 95-98 [KEK, report 79-18, 1979].

[7] M. Gell-Mann, P. Ramond and R. Slansky, Complex spinors and unified theories in Supergravity, P. van Nieuwenhuizen and D.Z. Freedman eds., North Holland Publishing Company, Amsterdam (1979), pg. 315-321. 
[8] S.L. Glashow, The future of elementary particle physics, in Quarks and leptons, proceedings of the Advanced Study Institute, Cargèse, Corsica (1979), M. Lévy et al. eds., Plenum, New York U.S.A. (1980), pg. 687.

[9] R.N. Mohapatra and G. Senjanović, Neutrino Mass and Spontaneous Parity Violation, Phys. Rev. Lett. 44 (1980) 912 [INSPIRE].

[10] G. Lazarides, Q. Shafi and C. Wetterich, Proton lifetime and fermion masses in an $\mathrm{SO}(10)$ model, Nucl. Phys. B 181 (1981) 287 [INSPIRE].

[11] R.N. Mohapatra and G. Senjanović, Neutrino Masses and Mixings in Gauge Models with Spontaneous Parity Violation, Phys. Rev. D 23 (1981) 165 [inSPIRE].

[12] J. Schechter and J.W.F. Valle, Neutrino masses in $\mathrm{SU}(2) \times \mathrm{U}(1)$ theories, Phys. Rev. D 22 (1980) 2227 [INSPIRE].

[13] E. Ma and U. Sarkar, Neutrino masses and leptogenesis with heavy Higgs triplets, Phys. Rev. Lett. 80 (1998) 5716 [hep-ph/9802445] [INSPIRE].

[14] D.-G. Lee and R.N. Mohapatra, Automatically $R$ conserving supersymmetric $\mathrm{SO}(10)$ models and mixed light Higgs doublets, Phys. Rev. D 51 (1995) 1353 [hep-ph/9406328] [InSPIRE].

[15] C.S. Aulakh, B. Bajc, A. Melfo, G. Senjanović and F. Vissani, The minimal supersymmetric grand unified theory, Phys. Lett. B 588 (2004) 196 [hep-ph/0306242] [INSPIRE].

[16] C.S. Aulakh, B. Bajc, A. Melfo, A. Rasin and G. Senjanović, SO(10) theory of R-parity and neutrino mass, Nucl. Phys. B 597 (2001) 89 [hep-ph/0004031] [InSPIRE].

[17] M.B. Einhorn and D.R.T. Jones, The Weak Mixing Angle and Unification Mass in Supersymmetric SU(5), Nucl. Phys. B 196 (1982) 475 [INSPIRE].

[18] W.J. Marciano and G. Senjanović, Predictions of Supersymmetric Grand Unified Theories, Phys. Rev. D 25 (1982) 3092 [inSPIRE].

[19] U. Amaldi, W. de Boer and H. Furstenau, Comparison of grand unified theories with electroweak and strong coupling constants measured at LEP, Phys. Lett. B 260 (1991) 447 [INSPIRE].

[20] J.R. Ellis, S. Kelley and D.V. Nanopoulos, Probing the desert using gauge coupling unification, Phys. Lett. B 260 (1991) 131 [INSPIRE].

[21] P. Langacker and M.-x. Luo, Implications of precision electroweak experiments for $m_{t}, \rho_{0}$, $\sin ^{2} \theta_{W}$ and grand unification, Phys. Rev. D 44 (1991) 817 [INSPIRE].

[22] C. Giunti, C.W. Kim and U.W. Lee, Running coupling constants and grand unification models, Mod. Phys. Lett. A 6 (1991) 1745 [inSPIRE].

[23] L. Du, X. Li and D.-X. Zhang, Proton decay in a supersymmetric $\mathrm{SO}(10)$ model with missing partner mechanism, JHEP 04 (2014) 027 [arXiv: 1312.1786] [INSPIRE].

[24] L. Du, X. Li and D.-X. Zhang, Connection between proton decay suppression and seesaw mechanism in supersymmetric SO(10) models, JHEP 1410 (2014) 36 [arXiv:1406.2081] [INSPIRE].

[25] M.B. Green and J.H. Schwarz, Anomaly Cancellation in Supersymmetric D $=10$ Gauge Theory and Superstring Theory, Phys. Lett. B 149 (1984) 117 [INSPIRE].

[26] M. Dine, N. Seiberg and E. Witten, Fayet-Iliopoulos Terms in String Theory, Nucl. Phys. B 289 (1987) 589 [INSPIRE]. 
[27] D. Wyler and L. Wolfenstein, Massless Neutrinos in Left-Right Symmetric Models, Nucl. Phys. B 218 (1983) 205 [INSPIRE].

[28] R.N. Mohapatra and J.W.F. Valle, Neutrino Mass and Baryon Number Nonconservation in Superstring Models, Phys. Rev. D 34 (1986) 1642 [InSPIRE].

[29] R.L. Arnowitt and P. Nath, Testing Supergravity Grand Unification at Future Accelerator and Underground Experiments, Phys. Rev. D 49 (1994) 1479 [hep-ph/9309252] [INSPIRE].

[30] B. Bajc, P.F. Perez and G. Senjanović, Minimal supersymmetric SU(5) theory and proton decay: where do we stand?, hep-ph/0210374 [INSPIRE].

[31] B. Bajc, G. Senjanović and F. Vissani, b- $\tau$ unification and large atmospheric mixing: a case for noncanonical seesaw, Phys. Rev. Lett. 90 (2003) 051802 [hep-ph/0210207] [INSPIRE].

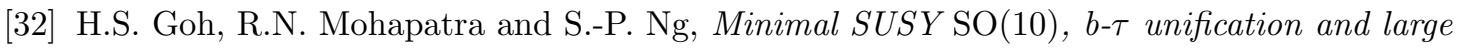
neutrino mixings, Phys. Lett. B 570 (2003) 215 [hep-ph/0303055] [INSPIRE].

[33] T. Fukuyama, A. Ilakovac, T. Kikuchi, S. Meljanac and N. Okada, Detailed analysis of proton decay rate in the minimal supersymmetric $\mathrm{SO}(10)$ model, JHEP 09 (2004) 052 [hep-ph/0406068] [INSPIRE].

[34] B. Bajc, A. Melfo, G. Senjanović and F. Vissani, The Minimal supersymmetric grand unified theory. 1. Symmetry breaking and the particle spectrum, Phys. Rev. D 70 (2004) 035007 [hep-ph/0402122] [INSPIRE].

[35] B. Bajc, A. Melfo, G. Senjanović and F. Vissani, Fermion mass relations in a supersymmetric SO(10) theory, Phys. Lett. B 634 (2006) 272 [hep-ph/0511352] [INSPIRE].

[36] S. Bertolini, T. Schwetz and M. Malinsky, Fermion masses and mixings in $\mathrm{SO}(10)$ models and the neutrino challenge to supersymmetric grand unified theories, Phys. Rev. D 73 (2006) 115012 [hep-ph/0605006] [INSPIRE].

[37] W. Grimus and H. Kühböck, Fermion masses and mixings in a renormalizable $\mathrm{SO}(10) \times Z_{2}$ GUT, Phys. Lett. B 643 (2006) 182 [hep-ph/0607197] [INSPIRE].

[38] G. Altarelli and G. Blankenburg, Different $\mathrm{SO}(10)$ paths to fermion masses and mixings, JHEP 03 (2011) 133 [arXiv: 1012.2697] [InSPIRE].

[39] A. Dueck and W. Rodejohann, Fits to SO(10) grand unified models, JHEP 09 (2013) 024 [arXiv:1306.4468] [INSPIRE].

[40] Z. Berezhiani and Z. Tavartkiladze, More missing VEV mechanism in supersymmetric SO(10) model, Phys. Lett. B 409 (1997) 220 [hep-ph/9612232] [INSPIRE].

[41] N. Sakai and T. Yanagida, Proton decay in a class of supersymmetric grand unified models, Nucl. Phys. B 197 (1982) 533 [InSPIRE].

[42] S. Weinberg, Supersymmetry at ordinary energies. Masses and conservation laws, Phys. Rev. D 26 (1982) 287 [INSPIRE].

[43] T. Fukuyama, A. Ilakovac, T. Kikuchi, S. Meljanac and N. Okada, General formulation for proton decay rate in minimal supersymmetric SO(10) GUT, Eur. Phys. J. C 42 (2005) 191 [hep-ph/0401213] [INSPIRE].

[44] C.S. Aulakh and S.K. Garg, The New Minimal Supersymmetric GUT : Spectra, RG analysis and Fermion Fits, Nucl. Phys. B 857 (2012) 101 [arXiv:0807.0917] [INSPIRE].

[45] C.S. Aulakh, NMSGUT-III: Grand Unification upended, arXiv:1107.2963 [INSPIRE]. 